\title{
Evaluación agroecológica de sistemas hortícolas de dos zonas del oriente antioqueño, Colombia
}

\section{Agroecological evaluation of horticultural systems of two zones in Eastern Antioquia, Colombia}
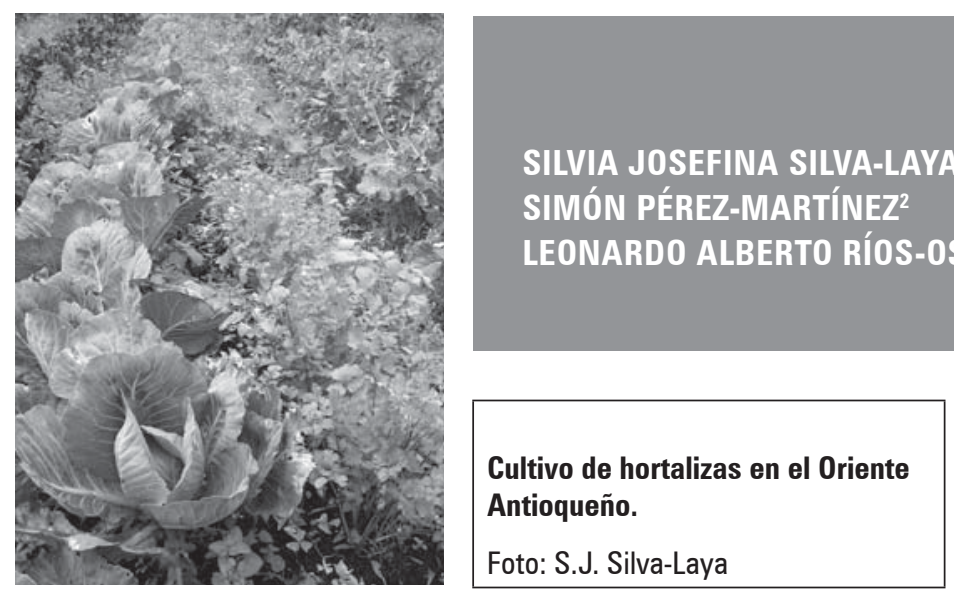

\section{RESUMEN}

La agroecología es un nuevo paradigma de la producción agrícola que se empieza a extender en las diferentes regiones con vocación agrícola de Colombia, sin embargo, se hace necesario conocer en detalle las características de estos sistemas y su nivel de sostenibilidad. El objetivo de este estudio fue evaluar la sostenibilidad de dos sistemas de producción agroecológica, principalmente de hortalizas (brócoli, cebolla, lechuga, col china, zanahoria, tomate, pepino, acelga, albahaca, perejil, cilantro, espinaca, ají, apio, calabacín, calabaza, entre otros) y frutales (aguacate, limón, tomate de árbol, mora y uchuva) en el oriente antioqueño de Colombia. Se diseñó un estudio observacional, descriptivo de tipo transversal entre marzo y octubre de 2013. Se calcularon y analizaron 20 indicadores de estado, a cada uno se le asignó un valor ponderado de 0 a 4 . En los resultados, ambas fincas mostraron valores similares, pero con diferencias en los indicadores B-Rendimiento y en la E-Densidad de Relaciones con Otros Colectivos. El factor más limitante en los índices de sostenibilidad general fue la falta de relevo generacional; así como la integración de una de estas experiencias con el entorno productivo de su respectiva localidad que se orienta por el modelo agrícola dominante basado en la revolución verde.

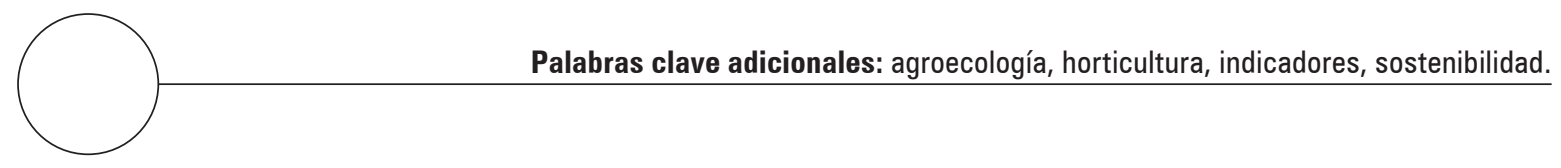

1 Programa de Doctorado en Agroecología, Universidad de Antioquia, Medellín (Colombia).

2 Facultad de Ciencias de la Ingeniería, Universidad Estatal de Milagro (UNEMI), Cantón Milagro, Guayas (Ecuador).

3 Grupo de Investigación Salud y Sostenibilidad, Escuela de Microbiología, Universidad de Antioquia, Medellín (Colombia).

4 Autor para correspondencia: leonardo.rios@udea.edu.co 


\section{ABSTRACT}

Agroceology is a new paradigm of agricultural production that starts to spread in different regions with agricultural vocation of Colombia, however, it is necessary to know in detail the characteristics of these systems and their level of sustainability. The aim of this study was to assess the sustainability of two systems of agro-ecological production, mainly vegetables (broccoli, onion, lettuce, Chinese cabbage, carrot, tomato, cucumber, chard, basil, parsley, coriander, spinach, chili pepper, celery, zucchini, pumpkin, etc.) and fruit (avocado, lemon, tree tomato, blackberry and cape gooseberry) in Eastern Antioquia in Colombia. An observational, descriptive cross-sectional study was designed between March and October 2013. There were calculated and analyzed 20 status indicators, each was assigned a weighted value from 0 to 4 . In the results, both farms showed similar values, but with differences in the B-Yield indicators and E-Density Relations with other groups. The most limiting in the rates of overall sustainability factor was the lack of generational change; and the integration of these experiences with the productive environment of their locality that is guided by the dominant agricultural model based on the green revolution.

Additional key words: agroecology, horticulture, indicators, sustainability.

Fecha de recepción: 03-09-2016 Aprobado para publicación: 24-11-2016

INTRODUCCIÓN

La agricultura convencional con base en los paquetes tecnológicos derivados a partir de la revolución verde se ha asociado con prácticas de monocultivos de alto rendimiento, mecanización y uso intensivo de productos agroquímicos (Altieri y Toledo, 2011). Esta revolución fue presentada como la opción adecuada de la agricultura para la producción de alimentos a gran escala $y$, por tanto, para mitigar el hambre a nivel mundial, frente a lo cual, diferentes autores han dado cuenta de la imposibilidad de alcanzar dicho objetivo a través de este modelo de agricultura (Altieri, 2009; Sarandón y Flores, 2014; Altieri y Nicholls, 2009a). El éxito económico y agrícola de estas técnicas ha contribuido a su establecimiento como un paradigma de producción, pero esta condición ha impedido hacer visible el impacto negativo de este modelo de producción sobre la salud humana y en la disposición de los recursos naturales en relación con la pérdida de biodiversidad, contaminación de acuíferos, degradación y salinización de suelos (Pengue, 2009; Nicholls et al., 2015).

Ante esta problemática emergen sistemas agrícolas de producción sostenible, los cuales se fundamentan en principios ecológicos y culturales, que en su conjunto reivindican el valor de la calidad de los alimentos producidos, la incorporación de distintas clases de conocimientos y saberes, la generación de tecnologías adaptadas al ambiente, la conservación de los recursos naturales y la promoción de procesos de producción con equidad y respeto en los planos económico, social y político para garantizar la autonomía y mejorar las condiciones de vida de los productores (León, 2012; Gliessman, 2013).

Los teóricos de este modelo de agricultura han propuesto principios ecológicos, económicos, socioculturales y políticos que los sistemas de producción deben cumplir para que sean considerados como sostenibles. En la dimensión ecológica, Altieri y Nicholls (2009) y Gliessman (2013) plantean la diversificación animal y vegetal en tiempo y espacio, el reciclaje de nutrientes y materia orgánica, la minimización de las pérdidas de suelo y agua manteniendo la cobertura del suelo, $\mathrm{y}$ el aprovechamiento de las sinergias que emergen de las interacciones planta-planta, planta-animal y animal-animal. Sevilla y Woodgate (2013) y Toledo y Barrera (2009) propusieron, en la dimensión sociocultural y política, romper con las formas de dependencia que ponen en peligro los mecanismos de reproducción, sean estas de naturaleza ecológica, socioeconómica o política por medio de la valoración de los conocimientos locales para su uso como elementos de creatividad y la mejora del nivel de vida de la población definida desde su propia identidad local. En la dimensión económica, Núñez (2010) planteó el establecimiento de circuitos cortos para el consumo de mercancías, que permitan una mejora de la calidad de 
vida de la población local y una progresiva expansión espacial, según los acuerdos participativos alcanzados por su forma de acción social colectiva.

En este sentido, autores como Flores et al. (2007), Giraldo y Valencia (2010), Pérez et al. (2005) y Blandi et al. (2013) han utilizado metodologías para evaluar diferentes sistemas de producción agrícola que toman en cuenta esta multidimensionalidad.

El oriente antioqueño de Colombia es una región con una temperatura media anual de $17^{\circ} \mathrm{C}$, precipitaciones anuales entre 1.800 y $2.000 \mathrm{~mm}$ y la humedad relativa es del $80 \%$. En ella se destina el $52 \%$ de su área a la producción agropecuaria primaria y abastece el 18\% del mercado agrícola colombiano (Lopera et al., 2011). El sector primario de la producción ha ocupado tradicionalmente el primer lugar en la región $(60 \%)$ (Lopera et al., 2011).

Históricamente en esta región, la producción agrícola ha estado asociada al minifundio ( $>80 \%$ de las fincas) y se ha caracterizado por ser altamente diversificada pues en cada parcela se cultivaba un promedio de cinco especies agrícolas en diferentes estadios de sucesión (Lopera et al., 2011). Sin embargo, la dinámica actual ha cambiado en dirección hacia los monocultivos de hortalizas y a la agroindustria de flores de exportación (Gobernación de Antioquia, 2009). La importancia agrícola de esta zona se ha sostenido por la creciente demanda de alimentos del área metropolitana de Medellín (Castaño, 2006).

Debido a que en años recientes en el oriente antioqueño se han gestado experiencias agroecológicas con diferentes niveles de desarrollo, el presente trabajo se propuso como objetivo evaluar la sostenibilidad de dos sistemas de producción agroecológica en esa zona.

\section{MATERIALES Y MÉTODOS}

Para la realización de esta investigación se diseñó un estudio observacional, descriptivo y transversal con un muestreo por conveniencia, en el cual las unidades de muestreo fueron dos de los 23 municipios del oriente antioqueño que, por su cercanía con el área metropolitana de Medellín, tenían mayor relevancia desde el punto de vista del abastecimiento de productos agrícolas a los mercados; los municipios seleccionados fueron Carmen de Viboral y Marinilla. En cada municipio se seleccionaron como unidades de análisis en el estudio, fincas catalogadas como agroecológicas o en proceso de conversión agroecológica por las asociaciones de productores locales; como criterio de exclusión se tuvo en cuenta la no aceptación de los dueños de las fincas para participar en el estudio. De acuerdo con estos criterios, en el municipio Carmen de Viboral (vereda La Milagrosa) se eligió la Finca Rena-Ser, y en el municipio Marinilla (vereda La Esmeralda) se eligió la Finca Las Brisas.

A partir de la aplicación de la matriz FODA se determinaron los criterios de diagnóstico y se derivaron 20 indicadores de estado a medir, vale decir, indicadores que aportan información referente a la situación del sistema en el momento del estudio (Sarandón y Flores, 2014) (Tab. 1). A cada uno de ellos se le asignó un valor ponderado de 0 a 4 (donde 0 representa el valor menos favorable a la sostenibilidad, 2 el valor umbral y 4 el valor óptimo). El estudio se realizó mediante la aplicación de una metodología híbrida validada por Silva y Pérez (2010) en la evaluación de sostenibilidad agroecológica de fincas productoras de durazno y otros frutales. Además, para calcular algunos indicadores del instrumento de evaluación se aplicaron metodologías de calidad de suelo y salud del cultivo (Nicholls y Altieri, 2002).

Para calcular los índices que miden el grado de cumplimiento de la dimensión ecológica, técnico-productiva (IE); socioeconómica (IK) y política-sociocultural (ISc) se les otorgó el mismo peso a cada uno de ellos y para el índice de sostenibilidad general (ISGen) se valoraron por igual las tres dimensiones. Así pues, los índices se calcularon con las siguientes fórmulas: $\mathrm{IE}=(\mathrm{A}+\mathrm{B}+\mathrm{C}+\mathrm{D}+(\mathrm{E} 1+\mathrm{E} 2) / 2+\mathrm{F}+\mathrm{G}) 7^{-1} ; \quad \mathrm{IK}=(\mathrm{A}+-$ $\mathrm{B}+\mathrm{C}+\mathrm{D}+\mathrm{E}+\mathrm{F}+\mathrm{G}) 7^{-1} ; \quad \mathrm{ISc}=[(\mathrm{A} 1+\mathrm{A} 2+\mathrm{A} 3) / 3+\mathrm{B}+-$ $\mathrm{C}+\mathrm{D}+\mathrm{E}+\mathrm{F}] 6^{-1}$ y ISGen $=(\mathrm{IK}+\mathrm{IE}+\mathrm{ISc}) 3^{-1}$; donde $\mathrm{A}$, $\mathrm{B}, \mathrm{C}, \mathrm{D}, \mathrm{E}, \mathrm{F}$ y $\mathrm{G}$ representan a los indicadores elegidos.

Para la evaluación de algunos indicadores se emplearon técnicas cualitativas como la entrevista en profundidad, encuestas cualitativas semiestructuradas y la observación no participante en campo. Con base en la aplicación de estos instrumentos se ponderaron ciertos atributos desde la perspectiva de los actores, para luego llevarlo a un valor estandarizado con el cual se realizaron los cálculos para determinar los índices de sostenibilidad ecológica, económica, sociocultural y general. 


\section{Tabla 1. Indicadores/subindicadores y sus valores estandarizados}

\begin{tabular}{|c|c|c|}
\hline \multicolumn{2}{|c|}{ Indicadores y subindicadores } & Estandarización de los indicadores \\
\hline & & Dimensión ecológica, técnico-productiva \\
\hline \multicolumn{2}{|l|}{ A-Calidad del suelo } & \multirow{2}{*}{$\begin{array}{l}\text { Se calcularon con la metodología calidad del suelo y salud del cultivo (Nicholls y Altieri, } \\
\text { 2002) }\end{array}$} \\
\hline \multicolumn{2}{|l|}{ B-Salud del cultivo } & \\
\hline \multicolumn{2}{|c|}{ C-Dependencia de insumos externos } & (4): Baja (de 0 a 33\%); (2): Media (34 a 67\%); (0): Alta (68 a 100\%) \\
\hline \multirow{2}{*}{$\begin{array}{l}\text { D-Manejo de } \\
\text { biodiversidad }\end{array}$} & D1-Diversidad temporal & $\begin{array}{l}\text { (4): Rota con otras especies. Deja parcela en barbecho un año o incorpora leguminosas } 0 \\
\text { abonos Verdes; (2): Realiza rotaciones eventualmente; (0): No realiza rotaciones }\end{array}$ \\
\hline & $\begin{array}{l}\text { D2-Diversidad espacial } \\
\text { de cultivos y vegetación } \\
\text { natural circundante } \\
\end{array}$ & $\begin{array}{l}\text { (4): } \geq 10 \text { especies cultivadas, parcelas rodeadas en al menos el } 50 \% \text { de sus bordes con } \\
\text { vegetación natural. (2): } 5 \text { especies de cultivos y rodeado al menos en un lado por vegetación } \\
\text { natural; (0): Monocultivo, rodeado de otros cultivos, campos baldíos o carretera }\end{array}$ \\
\hline \multicolumn{2}{|c|}{ E-Aplicación de prácticas tradicionales } & (4): Alta; (2): Media; (0): Baja \\
\hline \multicolumn{2}{|c|}{ F-Tecnologías endógenas adoptadas } & (4): Alta; (2): Media; (0): Baja \\
\hline \multicolumn{2}{|c|}{ G-Incidencia de plagas y enfermedades } & $\begin{array}{l}\text { (4): Alguna enfermedad o plaga en el último año que afectara menos del } 10 \% \text { de la pro- } \\
\text { ducción; (2): Alguna enfermedad o plaga en el último año, que afectó más del } 10 \% \text { de la } \\
\text { producción y menos de } 50 \% \text {; (0): Alguna enfermedad o plaga en el último año, que afectó } \\
\text { más del } 70 \% \text { de la producción }\end{array}$ \\
\hline \multicolumn{3}{|r|}{ Dimensión económica } \\
\hline \multicolumn{2}{|c|}{ A-Autosuficiencia alimentaria } & $\begin{array}{l}\text { (4): Produce al menos un rubro de cada grupo de alimentos }(\mathrm{I}, \mathrm{II}, \mathrm{III})^{1} ;(2) \text { : Produce al menos } \\
\text { un rubro de los grupos II y III; (0): Produce al menos un rubro del grupo III de alimentos) }\end{array}$ \\
\hline \multicolumn{2}{|l|}{ B-Rendimiento } & (4): Alta; (2): Media; (0): Baja \\
\hline \multicolumn{2}{|l|}{ C-Rentabilidad } & (4): Más del 20\%; (2): Entre 10 y 20\%; (0): Menos del 10\% \\
\hline \multicolumn{2}{|c|}{ D-Ingreso neto mensual } & (4): Más de dos salarios mínimos² (S.M); (2): Dos S.M.; (0): Menos de dos S.M. \\
\hline \multirow{2}{*}{$\begin{array}{l}\text { E-Riesgo } \\
\text { económico }\end{array}$} & $\begin{array}{l}\text { E1-Diversificación en la } \\
\text { venta }\end{array}$ & (4): Más de 7; (2): 4 a 6; (0): 1 a 3 \\
\hline & $\begin{array}{l}\text { E2-Número de vías de } \\
\text { comercialización. }\end{array}$ & $\begin{array}{l}\text { (4): Al menos una vía segura de comercialización en condiciones adversas; (2): } 3 \text { a 4, en } \\
\text { condiciones normales; (0): } 1 \text { o } 2 \text { en condiciones normales }\end{array}$ \\
\hline \multicolumn{2}{|c|}{ F-Prácticas de economía social y solidaria } & $\begin{array}{l}\text { (4): La mayoría de sus intercambios económicos son bajo esquemas de economía social } \\
\text { solidaria; } \\
\text { (2): Mantiene alguna relación de intercambio solidario con los vecinos; (0): No tiene relacio- } \\
\text { nes de intercambio con los vecinos }\end{array}$ \\
\hline & (4): Propietario; (2): Arrendatario más de 10 años; (0): Arrendatario menos de 5 años \\
\hline \multicolumn{2}{|c|}{ G-lenencia de la tierra } & Dimensión Sociocultural y Política \\
\hline \multirow{3}{*}{$\begin{array}{l}\text { A-Satisfacción de } \\
\text { las necesidades } \\
\text { básicas }\end{array}$} & A1-Vivienda & (4): Casa; (2): Rancho; (0): Habitación \\
\hline & $\begin{array}{l}\text { A2-Acceso a salud y } \\
\text { cobertura sanitaria }\end{array}$ & $\begin{array}{l}\text { (4): Centro de salud con médicos permanentes e infraestructura adecuada; (2): Centro de } \\
\text { salud con personal temporario medianamente equipado; (0): Sin centro de salud }\end{array}$ \\
\hline & A3-Servicios & $\begin{array}{l}\text { (4): Instalación de agua, luz, teléfono; (2): Sólo de agua; (0): Sin luz y sin fuente de agua } \\
\text { cercana }\end{array}$ \\
\hline \multicolumn{2}{|c|}{ B-Relevo generacional } & $\begin{array}{l}\text { (4): } 3 \text { o más descendientes o familiares trabajando en la finca; (2): } 2 \text { descendientes o fami- } \\
\text { liar trabajando en la finca; (0): Ningún familiar trabajando la finca }\end{array}$ \\
\hline \multicolumn{2}{|c|}{$\begin{array}{l}\text { C-Aceptabilidad del sistema de producción } \\
\text { (imaginarios ideológicos) }\end{array}$} & $\begin{array}{l}\text { (4): Está muy contento con lo que hace. No haría otra actividad aunque esta le reporte más } \\
\text { ingresos; (2): No está del todo satisfecho. Se queda porque es lo único que sabe hacer; } \\
\text { (0): Poco satisfecho con esta forma de vida. Anhela vivir en la ciudad y ocuparse de otra } \\
\text { actividad }\end{array}$ \\
\hline \multicolumn{2}{|l|}{ D-Cultura culinaria } & $\begin{array}{l}\text { (4): Consumo productos ecológicos (P.E.), locales, producidos en la comunidad; (2): Consumo } \\
\text { P.E. y no E. de la localidad; (0): No consumo P.E. ni locales }\end{array}$ \\
\hline \multicolumn{2}{|c|}{ E-Densidad de relaciones con otros colectivos } & (4): En más de un evento al mes; (2): En al menos un evento al mes; (0): Nunca participa \\
\hline \multicolumn{2}{|c|}{ F-Participación comunitaria } & $\begin{array}{l}\text { (4): Participa activamente en cada una de las organizaciones sociales; (2): Participa activa- } \\
\text { mente al menos en una organización; (0): No participa en ninguna organización }\end{array}$ \\
\hline
\end{tabular}

${ }^{1}$ Grupos de alimentos: I. Proteína animal, carne, huevo y leche. II. Legumbres, cereales, papa y yuca. III. Hortalizas y frutos.

${ }^{2}$ Salario mínimo: \$566.700. 


\section{RESULTADOS Y DISCUSIÓN}

Los productores de ambas experiencias han optado por la agroecología como forma para gestionar los recursos de sus predios. Los niveles de desempeño alcanzados por los indicadores medidos en cada experiencia fueron similares según la metodología empleada. Una visión general de las fincas se presenta en el tabla 2. El análisis de las relaciones entre los elementos de las fincas permitió diferenciar varios subsistemas en cada una de ella (Fig. 1).

\section{Análisis de los indicadores por dimensiones}

\section{Dimensión ecológica, técnico-productiva}

El promedio total del indicador A-Calidad del suelo fue de 3,55 en la finca Rena-Ser y de 3,64 en la finca Las Brisas, valores muy favorables a la sostenibilidad, con base en las técnicas rápidas de análisis de fertilidad de suelos propuesto por Nicholls y Altieri (2002) (Fig. 3A). En ambas experiencias se observó un suelo rico en materia orgánica, con presencia de diferentes organismos de micro y mesofauna, y mucha vegetación; permanece cubierto todo el año y utiliza abono orgánico que es producido dentro de los predios, con el reciclaje de desechos orgánicos de la finca. Todas estas características son favorables a la sostenibilidad, de acuerdo con autores como Abbona y Sarandón (2014); Altieri y Nicholls (2009b)
Adicional a las prácticas mencionadas, la rotación y asociación de cultivos y los policultivos; así como la existencia de barreras vivas, en Rena-Ser, contribuyen al buen desempeño del indicador B-Salud del cultivo (Fig. 3B) y la conservación de la vida del suelo, al mismo tiempo que disminuyen el riesgo de erosión.

La finca Las Brisas muestra mejor desempeño en el indicador B-Salud de los cultivos que la finca Rena-Ser en todos los subindicadores. La excepción fue el subindicador sistema de manejo (Fig. 3B), debido a que en la finca Rena-Ser se realizan mayores prácticas de conservación de suelo, tales como rotación y asociación de cultivos, parcelas en barbecho y cobertura vegetal; así como, un variado sistema de ciclaje de nutrientes que es muy importante para la conservación de la vida en el suelo (Altieri, 1999).

En cuanto al indicador C-Dependencia de insumos externos, el valor fue de 2 para ambas fincas. En Rena-Ser, entre 34 y $67 \%$ de los insumos se compra en el mercado, pues el productor utiliza concentrados comerciales para alimentar a los animales; asimismo, para la producción vegetal compra algunas plántulas de hortalizas y semillas como tomate. Para atender la producción agropecuaria el agricultor genera algunos insumos dentro del predio como el cultivo de pastos para contribuir a los subsistemas de compostaje y pecuario (Fig. 1).

La misma práctica se observó en el caso de Las Brisas; sin embargo este indicador mostró un valor medio

\section{Tabla 2. Características de las fincas estudiadas}

\begin{tabular}{|l|c|c|}
\hline \multirow{2}{*}{ Ubicación } & Rena-Ser & Las Brisas \\
\cline { 2 - 3 } & Municipio Carmen de Viboral, vereda La Milagrosa & Municipio Marinilla, vereda La Esmeralda \\
\hline \multirow{2}{*}{ Extensión } & $10.500 \mathrm{~m}^{2}$ & $3.200 \mathrm{~m}^{2}$ \\
\hline Temperatura & $2.100 \mathrm{~m}$ & $1.900 \mathrm{~m}$ \\
\hline No. de especies animales & $17^{\circ} \mathrm{C}$ & $17^{\circ} \mathrm{C}$ \\
\hline No. de especies vegetales & 1 & 3 \\
\hline Subsistemas identificados & 78 en 29 familias botánicas & 78 en 29 familias botánicas \\
\hline Índice de sostenibilidad general (ISGen) & 8 & 3,47 \\
\hline Índice ecológica, técnico-productiva (IE) & 3,59 & 3,60 \\
\hline Índice socioeconómica (IK) & 3,60 & 3,50 \\
\hline Política-sociocultural (ISc) & 4 & 3,33 \\
\hline Área de producción vegetal & 3,16 & $50 \%$ \\
\hline Área de producción animal animales & $36 \%$ & $50 \%$ \\
\hline Área de bosque y barbecho & $48 \%$ & $0 \%$ \\
\hline
\end{tabular}




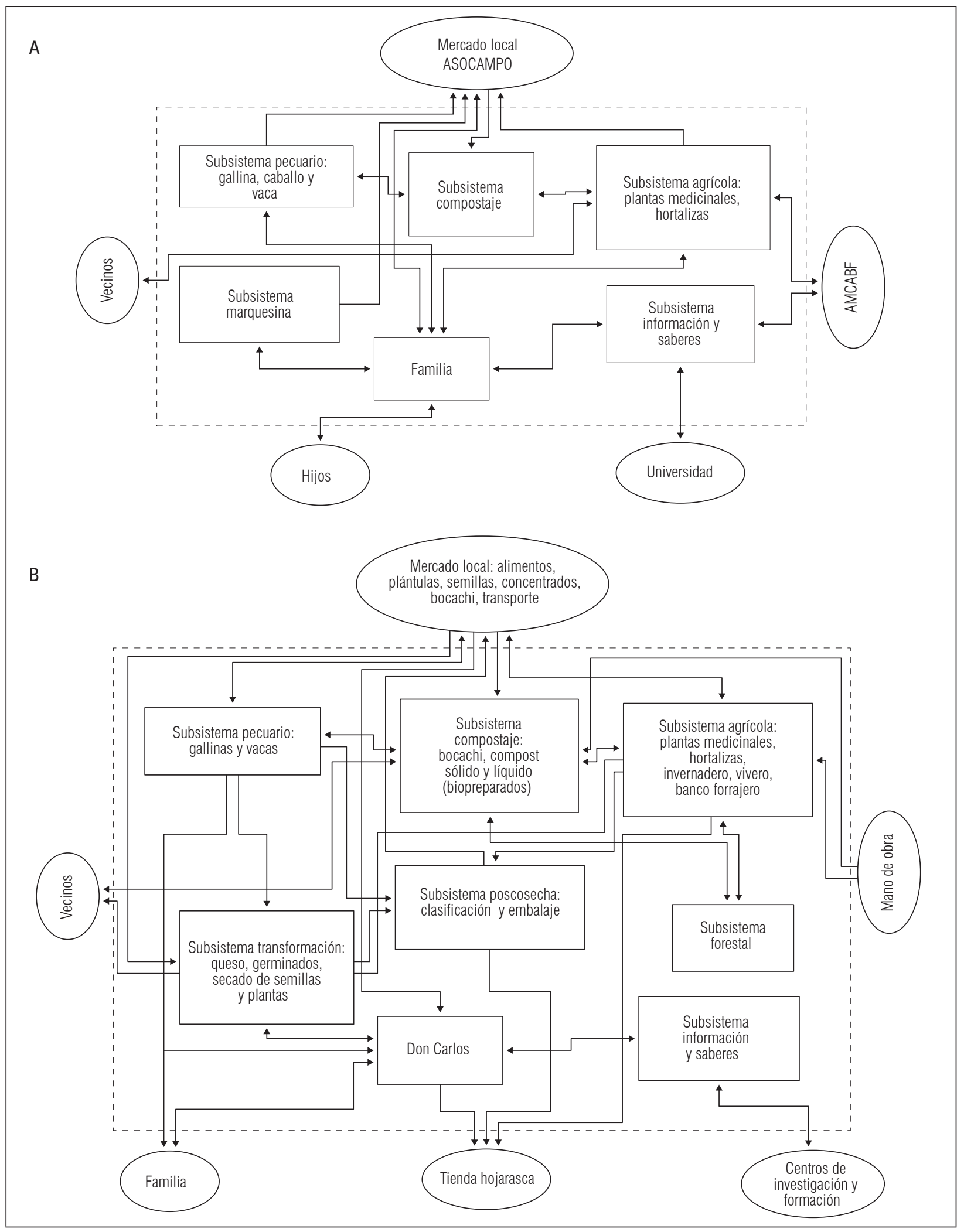

Figura 1. Subsistemas identificados en las Finca Rena-Ser (A) y Las Brisas (B). 


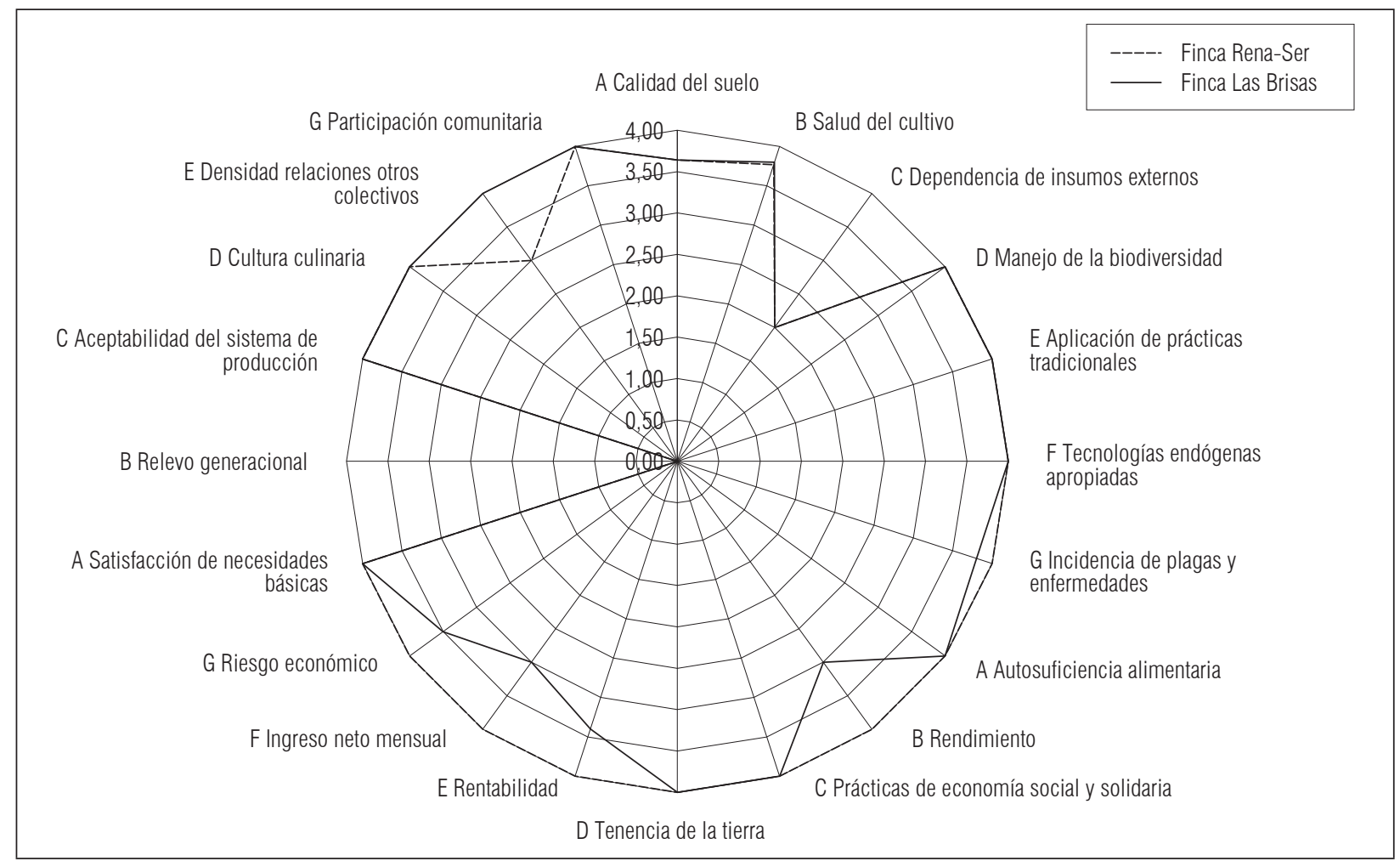

Figura 2. Niveles de desempeño para los indicadores de sostenibilidad de los sistemas de producción agrícola de las fincas Rena-Ser y Las Brisas.

debido a que los agricultores no producen dentro de la finca todos los insumos que necesitan para los subsistemas pecuario y agrícola (Fig. 1). Esta práctica de disminución de insumos externos a la finca favorece la autosuficiencia y, por tanto, mejora los indicadores de sostenibilidad ecológica y económica (Blandi et al., 2013)

Los indicadores E-Aplicación de prácticas tradicionales o reconstruidas y F-Tecnologías endógenas adoptadas, resultaron con valores óptimos a la sostenibilidad. La finca es autosuficiente en la fertilización del suelo y el manejo de plagas, pues tiene un sistema integrado de producción con el manejo de la biodiversidad funcional la cual, de acuerdo con Altieri y Nicholls (2009b) desempeña un papel decisivo en la sostenibilidad agroecológica de las fincas.

Este manejo les permite tener organismos con funciones ecológicas fundamentales porque mediante sus interacciones y sinergias optimizan los procesos del ecosistema, y permiten que este se autorregule, mejorando la eficiencia del uso de los recursos locales, como las plantas fijadoras de nitrógeno (leguminosas) (Paredes, 2013), aquellas que actúan como repelentes naturales (Nicotiana tabacum) y las plantas trampa como la col (Brassica oleracea var. capitata) (Vázquez et al., 2008; Van Driesche y Hoddle, 2007).

El indicador de D-Manejo de la biodiversidad, tuvo un valor óptimo para ambas experiencias. En Las Brisas, el inventario de biodiversidad vegetal (Tab. 3) mostró 78 tipos de plantas diferentes en 29 familias botánicas, con más de ocho tipos de usos y ubicadas en 7 subsistemas productivos de la finca. Dado que la finca tiene $3.200 \mathrm{~m}^{2}$, de los cuales aproximadamente $400 \mathrm{~m}^{2}$ son para producción de plantas medicinales y hortalizas, es importante resaltar la alta cantidad de especies y también de familias botánicas registradas, pues esto favorecerá la diversidad a nivel de finca, resultando en equilibrio las poblaciones de plagas y enfermedades o incluso su exclusión de acuerdo con lo que reporta la literatura (Altieri y Nicholls, 2009b; Vázquez et al., 2008; Vázquez y Martínez, 2015)

También es preciso reconocer la funcionalidad que cumplen las diferentes asociaciones de plantas, sobre todo la función de repelencia de plantas como ortiga (Urtiga dioica), caléndula (Calendula officinalis), ruda (Ruta graveolens), albahaca (Ocimum basilicum), 


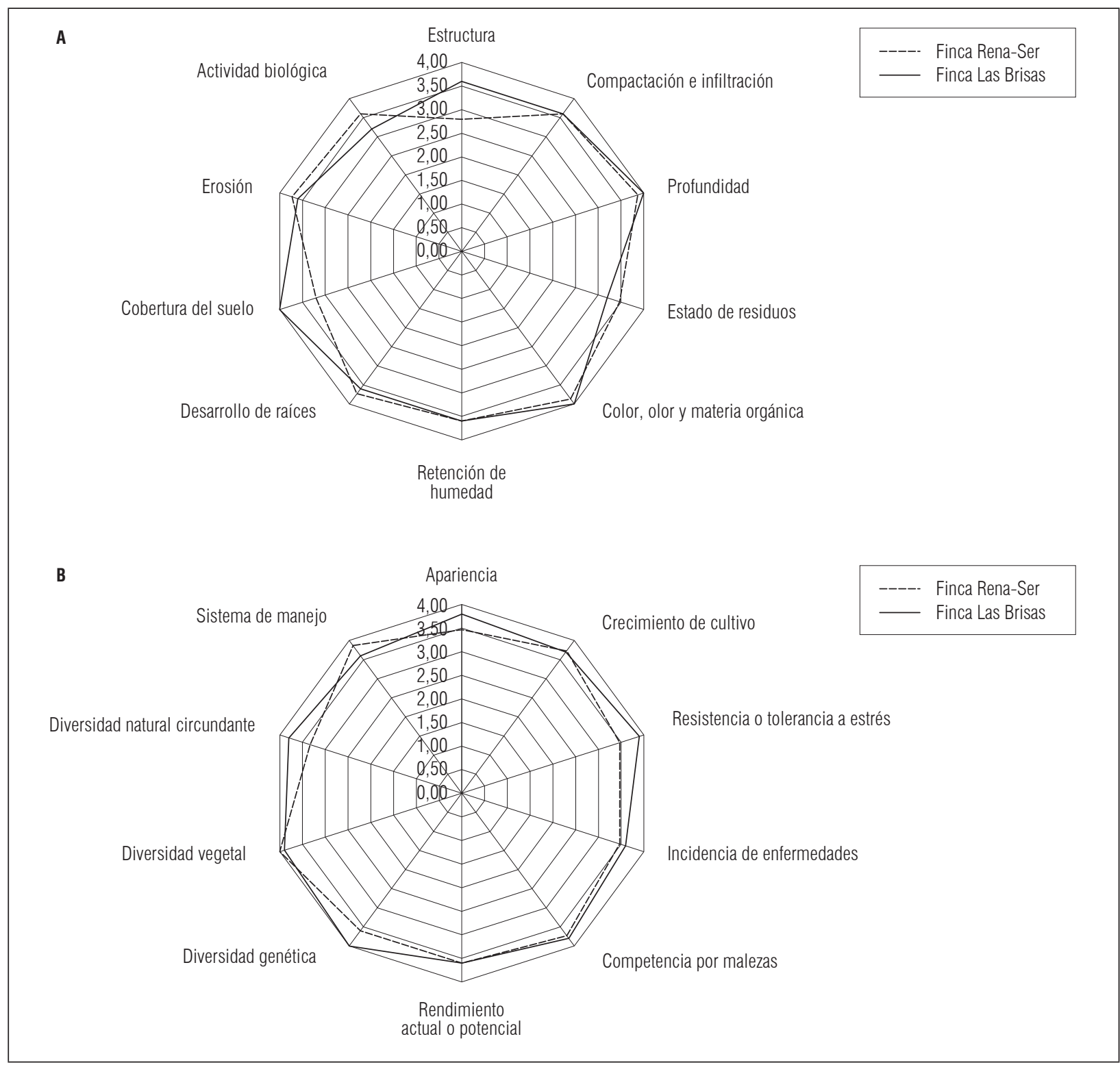

Figura 3. Comparación de indicadores y subindicadores de A-Calidad del suelo (arriba) y B-Salud de los cultivos (abajo) de las fincas Rena-Ser y Las Brisas.

cilantro (Eryngium foetidum), manzanilla (Chamaemelum nobile), romero (Rosmarinus officinalis), entre otras, intercaladas con las hortalizas. Muchas de estas especies presentaban flores (con polen y néctar) propicias para la alimentación de enemigos naturales, según refiere Nicholls (2008).

La finca Rena-Ser, con una superficie de $10.500 \mathrm{~m}^{2}$, posee alrededor de 78 especies vegetales (Tab. 3) y tres animales. Se observaron seis especies de árboles y arbustos asociadas al área de pastoreo, ya sea en el potrero o en los cercos vivos como: leguminosas como matarratón (Gliricidia sepium), chachafruto (Erythrina edulis); gramíneas y forrajeras como pasto kingrass (Pennisetum purpureum), maralfalfa (Pennisetum sp.), quiebrabarrigo (Trichantera gigantea) y botón de oro (Tithonia diversifolia). Árboles como guayabo (Psidium guajava), aguacate (Persea americana), laurel (Laurus nobilis), arrayán (Myrtus sp.), ciprés (Cupressus sp.), aliso (Alnus sp.) y acacia (Acacia sp) también fueron registrados.

Otra forma de aprovechamiento de la diversidad es su manejo en el espacio y en el tiempo mediante: 
Tabla 3. Número de especies cultivados en las fincas Rena-Ser (R) y Las Brisas (LB) según tipos y usos fundamentales

\begin{tabular}{|c|c|c|c|c|c|c|c|c|c|c|}
\hline \multirow{2}{*}{ Tipo/usos } & \multicolumn{2}{|c|}{ Alimento animal } & \multicolumn{2}{|c|}{ Ambiental } & \multicolumn{2}{|c|}{ Autoconsumo } & \multicolumn{2}{|c|}{ Venta } & \multicolumn{2}{|c|}{ Total general } \\
\hline & R & LB & $\mathrm{R}$ & LB & $\mathrm{R}$ & LB & R & LB & R & LB \\
\hline Árbol & & & 5 & 2 & 1 & & & & 6 & 2 \\
\hline Aromática & & & & & & 3 & & 7 & & 10 \\
\hline Fibra & & & & & & & & 1 & & 1 \\
\hline Forraje & & 1 & & & & & & & & 1 \\
\hline Frutal & & & & & 1 & 4 & 2 & 7 & 3 & 11 \\
\hline Hortaliza & & & & & 6 & 8 & 11 & 19 & 17 & 27 \\
\hline Medicinal & & & & & 33 & 9 & 10 & 3 & 43 & 12 \\
\hline Ornamental & & & 1 & 10 & & & & & 1 & 10 \\
\hline Pasto & 5 & & & & & & & & 5 & \\
\hline Raíces y tubérculos & & & & & & 2 & & 2 & 3 & 4 \\
\hline Total & 5 & 1 & 6 & 12 & 41 & 26 & 26 & 39 & 78 & 78 \\
\hline
\end{tabular}

1) La asociación simultánea, esto es la siembra en el mismo ciclo de otras especies benéficas o complementarias para el cultivo: col (Brassica oleracea)/cilantro, lechuga (Lactuca sativa)/cilantro; esta asociación en la mayoría de los casos es una siembra espontánea y natural, se produce porque quedan semillas de siembras anteriores, hacen equilibrio en ese ecosistema y, por ejemplo, se reproduce corazón herido (Polygonum nepalense), mostaza (Sinapis alba), manzanilla, entre otras. 2) La rotación de cultivos con hortalizas y leguminosa como col/arveja (Pisum sativum), haba (Vicia faba) o fríjol (Phaseolus vulgaris) intercalada entre cultivo y cultivo para recuperar los nutrientes del suelo en un ciclo; los sistemas de cultivo y manejo son siembra en mezcla o asociación simultánea y franjas alternadas, que se rotan cada dos ciclos.

En ambas unidades de análisis se ha implementado el manejo de la agrobiodiversidad como base para la estabilidad de los sistemas de producción para mejorar el manejo integrado de plagas y enfermedades, y eliminar riesgos de mercado.

\section{Dimensión socioeconómica}

El indicador A-Autosuficiencia alimentaria resultó con un valor muy favorable a la sostenibilidad en ambas experiencias, producen al menos un rubro de cada grupo de alimentos. Grupo I: leche, huevos y carne de gallina; grupo II (Tab. 1): fríjol, habas, arvejas, papas (Solanum tuberosum) y yuca (Manihot esculenta) y diversidad de hortalizas y grupo III: frutas como aguacate (Persea americana), limón (Citrus limón), lulo (Solanum quitoense), tomate de árbol (Cyphomandra betacea) y maracuyá (Passiflora edulis).

Los indicadores B-Rendimiento, E-Rentabilidad y F-Ingreso neto mensual resultaron con valores óptimos a la sostenibilidad en la finca Rena-Ser, de acuerdo con la escala utilizada en esta investigación. En el caso de Las Brisas, estos indicadores resultaron con menor valor pero por encima del umbral. Este es un beneficio de la diversidad que se maneja dentro de las fincas. El factor de la diversidad de la producción contribuye con el mantenimiento de una rentabilidad alta y un bajo riesgo económico pues se diversifican los ingresos; venden lo que producen en la finca y también obtienen ingresos adicionales cuando la finca recibe estudiantes (subsistema información y saberes; Fig. 1) que buscan aprender de su sistema de manejo. Estas son características propias de un sistema productivo con tendencias a la sostenibilidad económica, según reporta la literatura (Núñez, 2010; Razeto, 2000).

La finca Rena-Ser comercializa los productos, sin intermediarios, en la tienda Hojarasca a partir de prácticas de economía solidaria que se reflejan en la tienda, por la cooperación-asociación que ha logrado desarrollar en la comunidad. En la finca Las Brisas, comercializan la producción mediante la Asociación de Mujeres Campesinas Buscando Futuro (AMCABF) en la cooperativa Asocampo. 
El índice de sostenibilidad económica muestra un valor bastante favorable para Rena-Ser (4) y Las Brisas $(3,75)$; sin embargo, este solo es reflejo de una visión estática de los indicadores, pues si se relaciona con los indicadores de la dimensión sociocultural (relevo generacional) se evidencia fragilidad en el sistema, que incidiría negativamente sobre el resultado de la dimensión económica.

Es bueno resaltar la función de la economía solidaria en estas comunidades, para Razeto (2000) existe la necesidad de que la ciencia económica, no solo reconozca estatus teórico a las relaciones de intercambio $\mathrm{y}$ a los procesos por ella generados, sino que acepte en sus teorías el nivel micro y macroeconómico de la economía solidaria y campesina; así como, de sus relaciones y actividades.

\section{Dimensión sociocultural y política}

En ambas experiencias los resultados para los indicadores A-Satisfacción de las necesidades básicas, C-Aceptabilidad del sistema de producción y D-Cultura culinaria, fueron muy favorables a la sostenibilidad. En cuanto a asuntos de salud, el Sistema Subsidiado de Salud Gubernamental Básico (Sisbén) es el que los protege. Todos manifiestan no haber presentado enfermedades entre los miembros de la familia.

Al indagar sobre cómo perciben su calidad de vida, vinculada a la aceptación de su sistema de producción, todos respondieron que se encuentran muy satisfechos y que no cambiarían su actividad económica (agricultura campesina) por ninguna otra, ni su modo de producción agroecológica. Manifiestan estar muy contentos con lo que hacen y no se dedicarían a otra actividad aunque esta le reportara mayores ingresos económicos. Por esta razón, el indicador C-Aceptabilidad del sistema de producción resultó con un valor óptimo a la sostenibilidad en ambas fincas.

En lo referente al indicador D-Cultura culinaria, manifiestan consumir el 90\% de productos ecológicos de sus predios o producidos en la comunidad, respondiendo a sus tradiciones alimenticias y a la necesidad de consumir alimentos sanos, al mismo tiempo que cuidan el ambiente.

Respecto al indicador B-Relevo generacional, ambas experiencias resultaron con valores de cero, poco favorables a la sostenibilidad, en el trabajo de la finca solo intervienen los propietarios, sin ayuda familiar. La falta de relevo generacional obedece a la misma dinámica de migración en todo el municipio en los últimos 20 años, como lo mencionan Lopera et al. (2011).

Los indicadores G-Participación comunitaria y E-Densidad de relaciones con otros colectivos, resultaron con valores muy favorables a la sostenibilidad en ambos casos. En Las Brisas, la participación de la productora y de las mujeres campesinas de la zona en las diversas organizaciones del municipio ha beneficiado colectivamente a las mujeres campesinas de su vereda con el aporte de semillas, materiales para iniciar el cultivo, la máquina procesadora de plantas medicinales y aromáticas, la construcción de las marquesinas y los fogones eficientes.

Dentro de este marco, es bueno destacar que el índice de sostenibilidad política y sociocultural tuvo un valor de 3,1 para Las Brisas y de 3,6 para Rena-Ser, lo cual muestra un balance positivo en la satisfacción de los aspectos políticos y socioculturales. Pero el indicador que arrastra el índice hacia abajo es la ausencia de relevo generacional (en ambos casos), que pone en encrucijada la sostenibilidad del sistema en el tiempo.

El estudio de esta dimensión, en ambas experiencias, refleja la permanente interacción entre los sistemas biológicos y socioculturales de Marinilla, en el caso de Las Brisas y en el caso de Rena-Ser con las redes que ha establecido con asociaciones de productores, consumidores e investigadores tanto de la región, como a nivel nacional e internacional, en la cual el sistema ecológico evoluciona hasta reflejar su organización social, conocimientos, tecnologías y valores, como lo menciona.

\section{CONCLUSIONES}

Evaluar sistemas agroproductivos, con atención en las características específicas del sistema y en sus interrelaciones, permite entender la importancia de las sinergias para mantener el sistema en equilibrio, en producción constante, sin convertirse en una amenaza para la sostenibilidad.

Estos sistemas han alcanzado una producción eficiente a base de implementar prácticas de manejo de la biodiversidad funcional que le ayudan a tener organismos con funciones ecológicas fundamentales. La aplicación de estas prácticas les ha permitido la 
conservación del suelo, la poca incidencia de plagas y enfermedades, así como una dependencia media de insumos externos y un máximo aprovechamiento de los recursos internos para la producción agropecuaria en ambas fincas. Estas son un medio útil capaz de generar autosustento de manera constante y excedentes suficientes para participar en procesos de comercialización con prácticas de economía solidaria; revelando que desde la economía social se desarrollan estrategias productivas que permiten mejorar la calidad de vida. La articulación con diversos actores de la vida política, cultural y educativa les ha permitido a estos agricultores crecer y fortalecerse como organización.

\section{AGRADECIMIENTOS}

Agradecemos especialmente a Carlos Osorio, José Soto y Libia Giraldo por recibirnos en sus fincas y permitirnos la realización de este trabajo. A Ana Paula Neves, Carlos Martínez y Nancy Cardona por la colaboración en el levantamiento de la información.

\section{REFERENCIAS BIBLIOGRÁFICAS}

Abbona, E.A. y S.J. Sarandón. 2014. Manejo de nutrientes en los agroecosistemas. pp. 211-235. En: Sarandón, S.J. y C.C. Flores (eds.) Agroecología: Bases teóricas para el diseño y manejo de agroecosistemas sustentables. Universidad de La Plata, La Plata, Agentina.

Altieri, M.A. 1999. Agroecología, bases científicas para una agricultura sustentable. Nordan-Comunidad, Montevideo.

Altieri, M.A. 2009. Vertientes del pensamiento agroecológico: fundamentos y aplicaciones. SOCLA, Medellín. En: http//www.agroeco.org/socla; consulta: agosto de 2016.

Altieri, M. A. y C.I. Nicholls. 2009a. Cambio climático y agricultura campesina: impactos y respuestas adaptativas. LEISA Revista de Agroecología, marzo 2009, 5-8.

Altieri, M. y C. Nicholls. 2009b. Conversión agroecológica de sistemas convencionales de producción: teoría, estrategias y evaluación. Ecosistemas 16, 3-13.

Altieri, M. y V. Toledo. 2011. The agroecological revolution of Latin America: rescuing nature, securing food sovereignity. J. Peasant Studies 38, 587-612. Doi: 10.1080/03066150.2011.582947

Blandi, M., N. Gargoloff, C. Flores y S. Sarandón. 2009. Análisis de la sustentabilidad de la producción hortícola bajo invernáculo en la zona de La Plata, Argentina. Rev. Bras. Agroecol. (4)2, 1635-1638.

Blandi, M., M. Paleólogos, S. Sarandón e I. Veiga. 2013. Identificación de impedimentos para avanzar hacia una conducta sustentable en pequeños horticultores de La Plata, Argentina. Cuadernos Agroecol. 8(2), 1-5.

Castaño, I. 2006. Caracterización del desplazamiento forzado en el Oriente Antioqueño 1999-2005. Medellín. Corporación Región Oriente, Medellín, Colombia.

Flores, C., S. Sarandón y L. Vicente. 2007. Evaluación de la sustentabilidad en sistemas hortícolas familiares del partido de La Plata, Argentina, a través del uso de indicadores. Rev. Bras. Agroecol. 2(1), 180-184.

Giraldo, R. y F. Valencia. 2010. Evaluación de la sustentabilidad ambiental de tres sistemas de producción agropecuarios, en el corregimiento Bolo San Isidro, Palmira (Valle del Cauca). Rev. Investig. Agr. Ambient. (RIAA) 1(2), 7-17.

Gliessman, S. 2013. Agroecología: Plantando las raíces de la resistencia. Agroecol. 8(2), 19-26.

Gobernación de Antioquia. 2009. Plan estratégico por el desarrollo del oriente antioqueño (PLANEO). En: http:// planestrategicodeloriente.blogspot.com/2012/02/informe-final-planeo-view-more.html; consulta: abril de 2014.

León, T. 2012. Agroecología: La ciencia de los agroecosistemas - la perspectiva ambiental. IDEA, Universidad Nacional de Colombia, Bogotá.

Lopera, L., D. Salgado y R. Velázquez. 2011. ¿̇Es posible la agricultura orgánica en marinilla?: Entre la capacidad de los recursos y la voluntad política, se hace camino. Semestre Econ. 14, 135-152.

Nicholls, C. 2008. Control biológico de insectos: un enfoque agroecológico. Universidad de Antioquia, Medellín, Colombia.

Nicholls, C. y M. Altieri. 2002. Un método agroecológico rápido para la evaluación de la sostenibilidad de cafetales. Manejo Integrado Plagas Agroecol. 64, 17-24.

Nicholls, C., A. Henao y M. Altieri. 2015. Agroecología y el diseño de sistemas agrícolas resilientes al cambio climático. Agroecol. 10(1), 7-31.

Núñez, M. 2010. Venezuela ecosocialista: Un debate pendiente. Portatítulo, Caracas.

Pengue, W. 2009. Agricultura industrial y transnacionalización en América Latina. PNUMA, Buenos Aires.

Paredes, M. 2013. Fijación biológica de nitrógeno en leguminosas y gramíneas Trabajo Final de Ingeniería en Producción Agropecuaria. Facultad de Ciencias Agrarias, Pontificia Universidad Católica Argentina, Buenos Aires.

Pérez, B., C. González y L. García. 2005. Evaluación de la sustentabilidad de dos agroecosistemas campesinos de producción de maíz y leche, utilizando indicadores. Livestock Res. Rural Dev. 17(7).

Razeto, L. 2000. Desarrollo, transformación y perfeccionamiento de la economía en el tiempo. Universidad Bolivariana, Santiago de Chile. 
Sarandón, S.J. y C.C. Flores. 2014. Análisis y evaluación de agroecosistemas: Construcción y aplicación de indicadores. pp. 375-410. En: Sarandón, S.J. y C.C. Flores (eds.). Bases teóricas para el diseño y manejo de agroecosistemas sustentables. Universidad Nacional de La Plata, La Plata, Argentina.

Sevilla, E. y G. Woodgate. 2013. Agroecología: Fundamentos del pensamiento social agrario y teoría sociológica. Agroecol. 8(2), 27-34.

Silva, S. y S. Pérez. 2010. Sustentabilidad de fincas productoras de durazno en El Jarillo, Estado Miranda, Venezuela. RET. Rev. Estudios Transdiscipl. 2, 45-62.

Toledo, V. y N. Barrera. 2009. La memoria biocultural. Icaria, Barcelona, España.

Van Driesche, R. y M. Hoddle. 2007. El papel de la ecología de poblaciones y de los modelos de población en el control biológico. pp. 153-178. En: Forest Health Technology Enterprise Team: Control de plagas y malezas por enemigos naturales. En: www.socla.co/wp-content/uploads/2014/control-biologico-libroUCR.pdf?iv=74; consulta: agosto de 2016.

Vázquez, L. y H. Martínez. 2015. Propuesta metodológica para la evaluación del proceso de reconversión agroecológica. Agroecol. 10(1), 33-47.

Vázquez L, Y. Matienzo, M. Veitía y J. Alfonso. 2008. Conservación y manejo de enemigos naturales de insectos fitófagos en los sistemas agrícolas de Cuba. Instituto de Investigaciones de Sanidad Vegetal, Centro de Información y Documentación de Sanidad Vegetal (IDISAV), La Habana. 